\title{
Preparation and Properties of DMFC Membranes from Polymer- Brush Nanoparticles
}

\author{
Amir Khabibullin, Joanna J. Smith, Shelley D. Minteer and Ilya Zharov* \\ Departments of Chemistryand Materials Science and Engineering, University of Utah, Salt Lake City, Utah 84112
}

\begin{abstract}
We describe a novel approach for the preparation of proton conducting membranes using polymer brush nanoparticles (PBNPs). PBNPs can be assembled into the membranes directly, or the membranes can be prepared by first assembling porous scaffolds from unmodified NPs followed by surface modification with polymer brushes. In both cases, proton-conducting channels are formed in the interstitial spaces between the nanoparticles. First, proton conducting membranes were prepared using silica nanoparticles (SNPs) surface-grafted with 40-400 nm sulfonated polymer brushes, poly(3sulfopropylmethacrylate), pSPM, and poly(4-styrenesulfonic acid), pSSA, grown via surface initiated atom transfer radical polymerization (SI-ATRP). The membranes prepared from the NPs carrying longer polymer chains possessed polymer-like characteristics, compared to the stiffer membranes made with shorter polymer brushes. All these membranes showed comparable proton conductivities, with a maximum value of $\sim 0.06 \mathrm{~S} / \mathrm{cm}$ at $98{ }^{\circ} \mathrm{C}$ and $70 \%$ R.H. Second, we prepared proton conducting pore-filled membranes. In this case, nanoporous colloidal crystals were first assembled from unmodified SNPs, followed by filling the pores with PSPM or pSSA brushes covalently attached to the pore surface using SIATRP. The membranes could be hydrated almost completely, and did not swell. The proton conductivity was similar for both polymer-filled membranes, with a maximum value of $\sim 0.02 \mathrm{~S} / \mathrm{cm}$ achieved at $30{ }^{\circ} \mathrm{C}$ and $94 \%$ R.H. A sigmoidal dependence of the proton conductivity on the amount of sulfonic acid groups was found for pSPM pore-filled membranes. The proton conductivity remained relatively low at low degrees of sulfonation, increased rapidly around $50 \%$ sulfonic acid group content, and did not increase significantly after reaching ca. $75 \%$ sulfonic acid group content. We found that OCV for DMFC MEAs built using these membranes reached its maximum at $65 \%$ sulfonic group, and decreased after that. We attribute this effect to the increased methanol cross-over, which was confirmed by methanol uptake measurements for the membranes.
\end{abstract}

\section{Introduction}

The preparation and study of proton conductive materials that are the key component of the fuel cell ${ }^{1}$ is of high importance. Fuel cell membranes have to possess high proton conductivity, be mechanically and thermally stable, remain hydrated at high temperatures, ${ }^{2,3}$ should be nonswelling in methanol and water and possess low methanol permeability. ${ }^{4}$ For nearly all known PEM materials heating leads to decreased proton conductivity due to the water removal and decreased ion solvation. In addition, heating leads to the loss of connectivity among ion-rich clusters that exist in most polymeric PEM materials, which also results in the drop in conductivity. ${ }^{5-7}$ Methanol permeation and swelling in methanol and water ${ }^{8}$ also limit the fuel cell efficiency.

To overcome these limitations, thermally stable and nonswelling membranes with high proton conductivity over a wide temperature range are desirable. ${ }^{9}$ Polymer electrolyte membranes (PEMs) are the most commonly used proton conducting materials, ${ }^{10}$ but they do not satisfy all of these requirements. A novel approach to proton conductive materials is to prepare organic-inorganic composites $^{11,12}$ usinginorganic oxide nanoparticles dispersed inside a polyelectrolyte. We decided to explore another approach to hybrid proton-conducting membranes, using polymer brush nanoparticles (PBNPs) in two ways. We self-assembled PBNPs carrying sulfonated polymer brushes into the proton conducting membranes. This method is particularly simple, and it leads to materials with high proton conductivity and water uptake. Alternatively, we assembled silica nanoparticles (SNPs) into colloidal membranesthat served as a mechanically stable matrix containing a continuous network of nanopores, and porefilled these membranes with surface-graftedsulfonated polymer brushes. The SNP matrix allows studying proton transport though nanoporous materials and achieving high degrees of polymer sulfonation, otherwise impossible to achieve due to the formation of water soluble polymers.

We compared the pore-filled and self-assembled PBNP membranes and studied their water uptake, proton conductivity and fuel cell performance as a function of sulfonation extent, as described below. 


\section{Experimental}

Materials. Tetraethyl orthosilicate (TEOS) (99+\%, Alfa Aesar) was used as received. 3-Sulfopropylmethacrylate, potassium salt (SPM) (98\%), 4-Styrenesulfonic acid, sodium salt (SSA), 3Aminopropyltriethoxy-silane (APTES) (99\%), 2-Bromoisobutyryl bromide (98\%), Copper(I) chloride (99.995+\%), Copper(II) chloride (99.999\%), Copper(I) bromide (99.999\%), 2,2'Dipyridyl (Bipy) (ReagentPlus $\geq 99 \%$ ), and tetrabutylammonium hydroxide (40 wt\% solution in water) were obtained from Aldrich and used as received. 4-Dimethyl-aminopyridine (DMAP) $(\geq 98.0 \%$, Fluka) was used as received. Silver Print II (GC Electronics) was used to coat the colloidal membranes for EIS measurements (see below).

Instrumentation. Dynamic light scattering (NICOMP 380 ZLS) was used to determine the size of polymer-modified silica particles. Scanning electron microscope (Hitachi S300N) and tunneling electron microscope (FEI Techna G2 T-12) were used to image unmodified and polymer-modified silica particles and pore-filled membranes. Nuclear magnetic resonance NMR (Varian I-500) was used to determine the structure and composition of copolymers in solution. Gel permeation chromatography (AKTA FPLC) was used to determine the molecular weight of copolymers in solution. Thermogravimetric analysis (TGA) of polymer-modified silica particles was performed using TGA Q500 (TA Instruments). Nuclear magnetic resonance NMR (Varian I-500) was used to determine the structure and composition of copolymers in solution. Gel permeation chromatography (AKTA FPLC) was used to determine the molecular weight of copolymers in solution.

The complex impedance of the samples was measured using Princeton Applied Research VersaSTAT. Silver paint was coated on both sides of the membranes to serve as electrodes. The impedance was measured using a two-probe testing device placed in humidity- and temperature-controlled chamber. The complex impedance of the samples was measured and the proton conductivity was calculated using $\sigma=1 / \mathrm{RA}$, where $\sigma$ is the ionic conductivity, $\mathrm{l}$ is the distance between the two electrodes, $\mathrm{R}$ is the ohmic resistance of the membrane, $\mathrm{A}$ is the cross-sectional area of the material.

OCV and linear polarization measurements were carried out using DY-2023 bipotentiostat (Digi-IVY) in a home-made static membrane-electrode assembly (MEA) with pore-filled silica colloidal membranes.The $10 \times 10 \mathrm{~mm}$ square platinized carbon cloth (ELAT GDE $5 \mathrm{gpm}$ ) was used as the cathode. The anode was prepared using the catalyst powder (20\% HP Pt:Ru alloy (1:1 atomic ratio) on Vulcan XC-72R Carbon, which was suspended in water-ethanol mixture (4 $\mathrm{mg}$ of powder alloy in $50 \mu \mathrm{L}$ water and $100 \mu \mathrm{L}$ ethanol) and evenly applied using paint brush onto 4 square $(10 \times 10 \mathrm{~mm})$ pieces of Toray paper, resulting in $1 \mathrm{mg} / \mathrm{cm}^{2}$ of the catalyst on Toray paper. The resulting pieces were air dried for 12 hours and were ready to use. Anode and cathode were placed inside custom made $21 \times 15 \mathrm{~mm}$ graphite plates which had 4 loops of serpentine channel of $0.8 \mathrm{~mm}$ width and $11 \mathrm{~mm}$ length for each loop and were modified with slits to inject fuel. The plates were attached to the pore-filled silica membranes using doublesided tape. The aluminum foil strips were clamped to graphite plates using a plastic clamp and connected to potentiostat with alligator clips. $3 \mathrm{M}$ methanol solution in water was used as fuel, which was supplied drop wise to the anode using a syringe through the slits in graphite plates. The cathode was exposed to the air which served as a source of oxygen.

The average water uptake of the polymer-modified membranes was calculated as follows:

$$
\text { water uptake }(\%)=\frac{W_{w e t}-W_{d r y}}{W_{d r y}} \times 100 \% \text { (1) }
$$
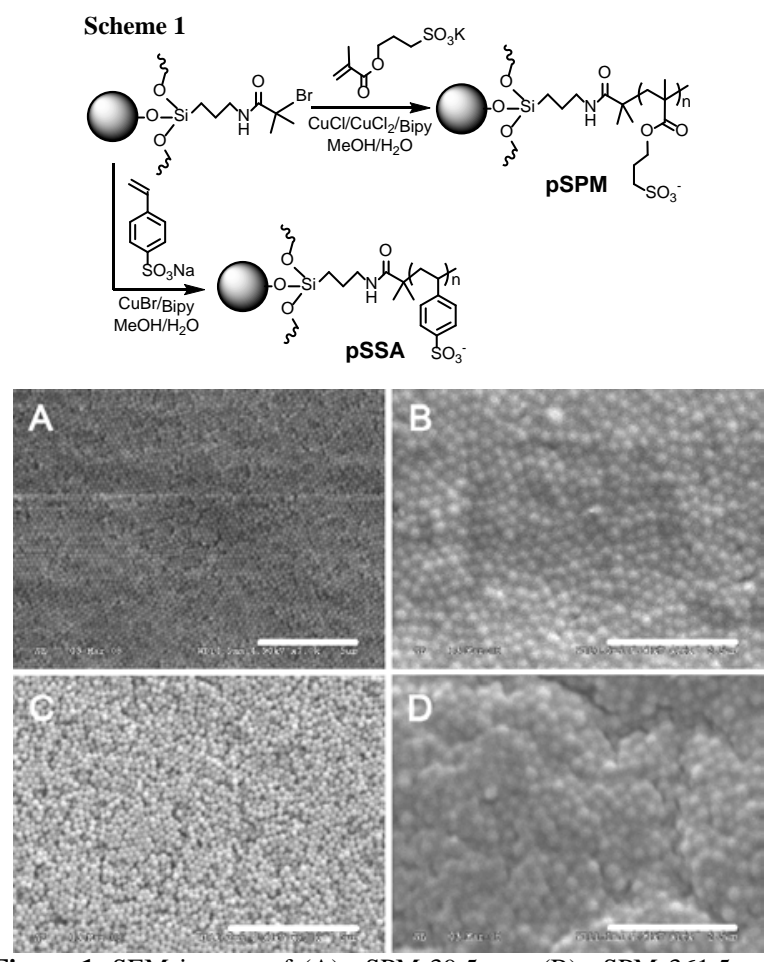

Figure 1. SEM images of (A) pSPM-39.5 nm, (B) pSPM $361.5 \mathrm{~nm}$, (C) pSSA-41.5 nm, and (D) pSSA-486 nm polymer-modified selfassembled silica membranes. Size bars: A, C $=5 \mu \mathrm{m}, \mathrm{B}, \mathrm{D}=2.5 \mu \mathrm{m}$.

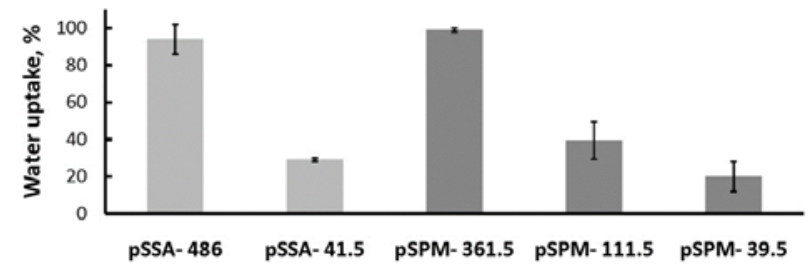

Figure 2. Water uptake of self-assembled PBNP membranes after keeping membranes in a humidity-temperature controlled chamber at $97 \%$ R.H. and room temperature for 24 hours.

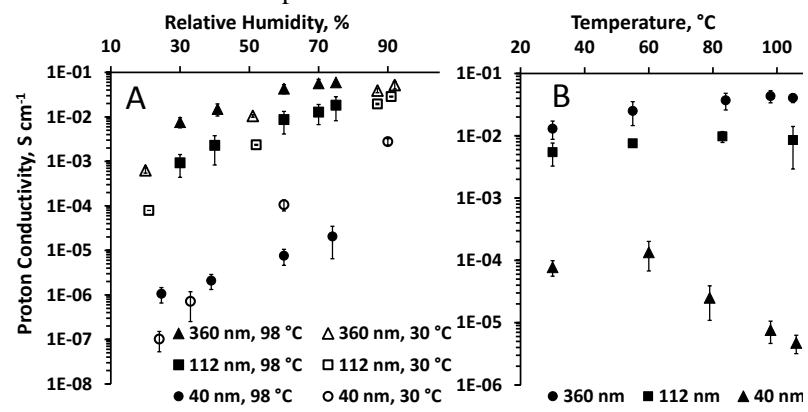

Figure 3. Proton conductivity of pSPM membranes as a function of (A) relative humidity at $30^{\circ} \mathrm{C}$ and $98{ }^{\circ} \mathrm{C}$ and (B) temperature at $60 \%$ relative humidity.

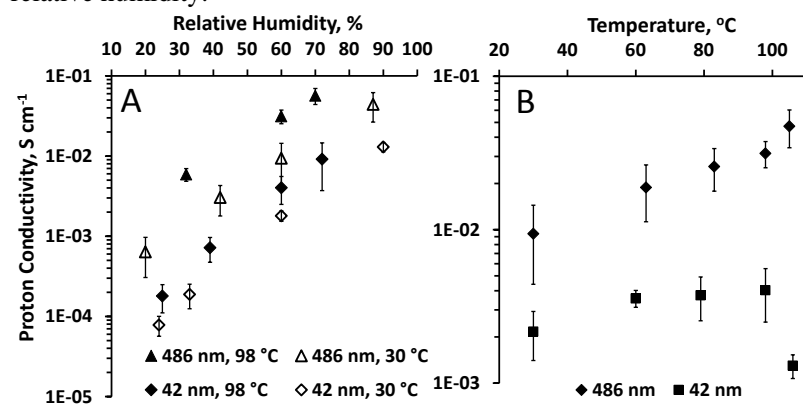

Figure 4. Proton conductivity of pSSA membranes as a function of (A) relative humidity at $30{ }^{\circ} \mathrm{C}$ and $98{ }^{\circ} \mathrm{C}$ and (B) temperature at $60 \%$ relative humidity. 
where $W_{\text {dry }}$ is the dry weight of the membrane and the $\mathrm{W}_{\text {wet }}$ is the wet weight of the membrane.The methanol uptake of the porefilled membranes was calculated as follows:

$$
\text { methanol uptake }(\%)=\frac{W_{M e O H}-W_{d r y}}{W_{d r y}} \times 100 \% \text { (2) }
$$

where $W_{\text {dry }}$ is the dry weight of the membrane and $W_{\mathrm{MeOH}}$ is the weight of the membrane after soaking in methanol.

Preparation PBNPs. Silica spheres were synthesized using the Stöber method. ${ }^{13}$ Poly(3-sulfopropylmethacrylate), pSPM and poly(stryrene-sulfonic acid), pSSA, brushes were grown onto initiator-modified silica spheres via atom transfer radical polymerization (ATRP). First, the silica nanoparticles $(1 \mathrm{~g})$ were suspended in $50 \mathrm{~mL}$ of $0.06 \mathrm{M}$ 3-aminopropyltriethoxysilane (APTES) dry acetonitrile solution for 17 hours at room temperature. The amine-functionalized particles $(1 \mathrm{~g})$ were then suspended in $50 \mathrm{~mL}$ of dry dichloromethane solution of $0.15 \mathrm{M}$ dry triethylamine, $0.13 \mathrm{M}$ 2-bromoisobutyryl bromide, and a catalytic amount of DMAP for 12 hours at room temperature. The grafting of pSPM brushes onto the initiator-modified silica spheres ( $1 \mathrm{~g}$ ) was carried out in $10 \mathrm{~mL}$ of a 2:1 mixture of degassed methanol and water, containing 3-sulfopropylmethacrylate potassium salt $(0.5 \mathrm{M}), 2,2$ '-dipyridyl $(0.08 \mathrm{M})$, $\mathrm{CuCl}_{2}(0.006 \mathrm{M})$, and $\mathrm{CuCl}(0.02 \mathrm{M})$ at room temperature. Similarly, the polymerization of pSSA brushes onto initiatormodified silica spheres were carried out in a 2:3 mixture of degassed methanol and water, containing styrenesulfonic acid sodium salt (0.8 M), 2,2'-dipyridyl (0.03 M), and $\mathrm{CuBr}(0.02 \mathrm{M})$ at room temperature. The polymerization times were varied from $20 \mathrm{~min}$ to $24 \mathrm{~h}$.

Preparation of Self-Assembled PBNP Membranes. Silica colloidal membranes were prepared by casting of the polymermodified silica spheres from a $\sim 4 \mathrm{wt} \%$ ethanol colloidal solutions followed by solvent evaporation. This process resulted in the formation of $\sim 200 \mu \mathrm{m}$ thin membranes.

Preparation and Modification of Sintered PBNP Membranes. Sintered silica colloidal membranes were prepared as reported earlier. ${ }^{14,15}$ The thickness of the membrane varied from 0.9 to $1.5 \mathrm{~mm}$. The sintered membranes were rehydroxylated in a solution of tetrabutylammonium hydroxide of $\mathrm{pH} 9.5$ at $60^{\circ} \mathrm{C}$ for $48 \mathrm{~h}$ and were modified with amines by placing the rehydroxylated sintered membranes in $10 \mathrm{~mL}$ of $0.05 \mathrm{M}$ APTES dry acetonitrile overnight at room temperature under $\mathrm{N}_{2}$ (g). The amine-modified sintered membranes were then placed in $10 \mathrm{~mL}$ of dry dichloromethane solution of $0.15 \mathrm{M}$ dry triethylamine, 0.13 M 2-bromoisobutyryl bromide, and a catalytic amount of DMAP and allowed to react overnight at room temperature. The sulfonated polymers were then introduced to the initiatormodified silica membranes via ATRP. The polymerization of pSPM was carried out in a 2:1 mixture of degassed methanol and water, containing 3-sulfopropyl-methacrylate potassium salt $(0.7$ M), 2,2'-dipyridyl (0.03 M), $\mathrm{CuCl}_{2}(0.003 \mathrm{M})$, and $\mathrm{CuCl}(0.007$ M) at room temperature under $\mathrm{N}_{2}$ (g) for $\sim 10 \mathrm{hrs}$. Similarly, the polymerization of pSSA brushes onto initiator-modified silica spheres were carried out in a 2:3 mixture of degassed methanol and water, containing styrenesulfonic acid sodium salt $(0.8 \mathrm{M})$, 2,2'-dipyridyl (0.03 M), and $\mathrm{CuBr}(0.02 \mathrm{M})$ at room temperature under $\mathrm{N}_{2}$ (g) for 10 hrs.

\section{Results and discussion}

Self-Assembled PBNP Membranes. Sulfonated linear polymer brushes, pSPM and pSSA, were grown onthe initiator-grafted silica nanoparticles via ATRP (Scheme 1). The length of these bushes was controlled by the polymerization time in the range of $40-400 \mathrm{~nm}$, and monitored by DLS. Thermograviametric analysis was also

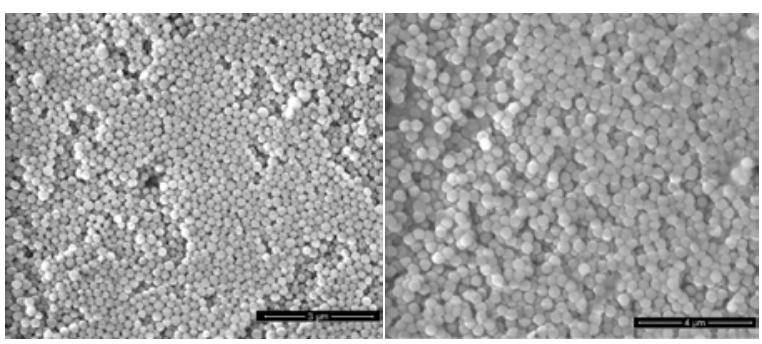

Figure 5. SEM images of self-assembled (left, scale bar is $3 \mu \mathrm{m}$ ) and pressed (right, scale bar is $4 \mu \mathrm{m}$ ) sintered silica colloidal membranes pore-filled with PSPM/PEEMA brushes (50 mol\% SPM).

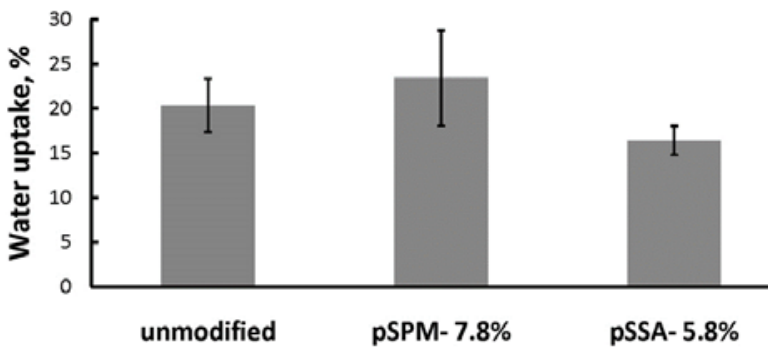

Figure 6. Water uptake for sintered silica membranes after soaking in water at room temperature for 24 hours.

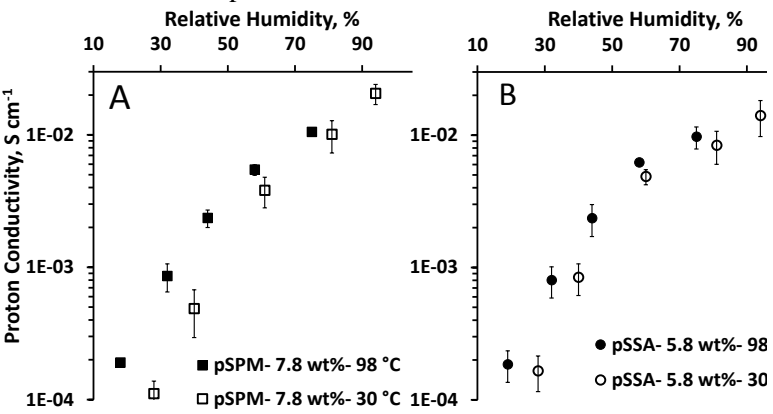

Figure 7. Proton conductivity of (A) pSPM- and (B) pSSA-filled sintered colloidal membranes as a function ofrelative humidity at 30 ${ }^{\circ} \mathrm{C}$ and $98^{\circ} \mathrm{C}$.

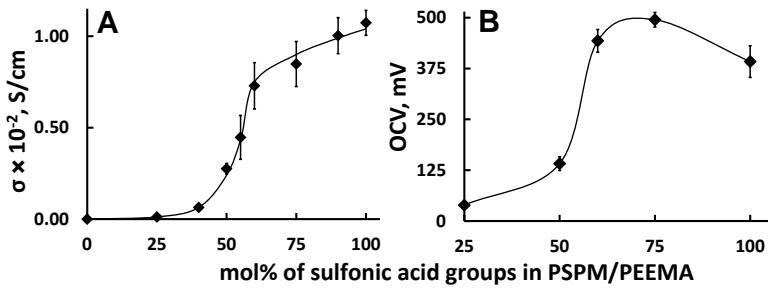

Figure 8.(A) Plot of room temperature proton conductivity (at $98 \%$ R.H.) and (B) room temperature open circuit voltage for pore-filled membranes as a function of SPM content in the pore-filling copolymer.

performed for the resulting polymer brush nanoparticles (PBNPs). The surface-grafted pSSA began to decompose around $250^{\circ} \mathrm{C}$, while the pSPM brushes were less stable and started to decompose around $150{ }^{\circ} \mathrm{C}$. The \%wt loss due to the polymer for pSPM-grafted particles was $2.7 \%$ after 20 min of polymerization, $10.6 \%$ after 2 hours of polymerization, and $35.8 \%$ after 6 hours of polymerization. Particles grafted with pSAA brushes for 1 hour lost $4.5 \%$ of their weight, while those grafted for 24 hours lost $22.2 \%$ of their weight. This further demonstrated that longer polymerization times lead to the growth of longer polymer chains.

The membranes prepared by casting of the PBNPs carrying short polymer brushes were stiff. Their SEM 
showed the individual silica nanoparticles and nanopores (Figure 1A, C).Casting of the silica spheres carrying longer polymer chains (Figure 1B, D) resulted in polymer-like films. These membranes were flexible, compared to the stiff membranes made with shorter polymer brushes.

The membranes were kept in a constant humiditytemperature chamber at $97 \%$ R.H. and room temperature for $24 \mathrm{~h}$ and their average water uptake were calculated using equation (1). The water uptake increased with increasing polymer brush length and reached nearly $100 \%$ forthe membranes made using PBNPs with long polymer brushes (Figure 2).

For both types of self-assembled PBNP membranes, pSPM and pSSA, there is an increase in proton conductivity with longer polymer brushes (Figures $3 \mathrm{~A}$ and $4 \mathrm{~A}$ ), due to an increase in the number of sulfonic acid groups. The membranes possess comparable proton conductivities, with a maximum value of $\sim 0.06 \mathrm{~S} \mathrm{~cm}^{-1}$ for both pSPM $362 \mathrm{~nm}$ and pSSA $486 \mathrm{~nm}$ at $98{ }^{\circ} \mathrm{C}$ and $70 \%$ R.H. As expected, the proton conductivity of all the membranes increases with increasing relative humidity. At $30{ }^{\circ} \mathrm{C}$ and $\sim 20 \%$ R.H., the proton conductivity of $362 \mathrm{~nm}$ pSMP and $486 \mathrm{~nm}$ pSSA membranes was $0.0006 \mathrm{~S} \mathrm{~cm}^{-1}$. At $30{ }^{\circ} \mathrm{C}$ and $\sim 90 \%$ R.H., the proton conductivity of 362 $\mathrm{nm}$ pSMP and $486 \mathrm{~nm}$ pSSA membranes was 0.052 and $0.044 \mathrm{~S} \mathrm{~cm}^{-1}$, respectively.

The proton conductivity for most of the membranes increases with temperature (Figure 3B and 4B), except for the pSPM $40 \mathrm{~nm}$ and pSSA $42 \mathrm{~nm}$ membranes. These membranes possess lower conductivity at higher temperature. We speculate that this is the result of a short length of the polymer chains, which makes it hard to retain water molecules at higher temperatures. At $30{ }^{\circ} \mathrm{C}$ and $70 \%$ R.H., the proton conductivity of $362 \mathrm{~nm}$ pSMP and $486 \mathrm{~nm}$ pSSA membranes was $0.028 \mathrm{~S} \mathrm{~cm}^{-1}$ and $0.019 \mathrm{~S} \mathrm{~cm}^{-1}$, respectively. The conductivity increased to $0.057 \mathrm{~S} \mathrm{~cm}^{-1}$ at $98{ }^{\circ} \mathrm{C}$ and $70 \%$ R.H. for both $362 \mathrm{~nm}$ pSPMand486 nm pSSAmembranes.

Pore-Filled PBNP Membranes.First, silica colloidal membranes were assembled by vertical deposition or by pressing SNPs in a die set, followed by sintering, as described in the Experimental section.Next, the sintered silica membranes were pore-filled with pSPM and pSSA sulfonated polymer brushes by surface-initiated ATRP. SEM images of the pore filled membranes are shown in Figure 5. The TGA\%wt loss for pSPM membranes leveled off at $\sim 8 \mathrm{wt} \%$ and the pSSA membranes leveled off at $\sim 6$ wt $\%$ after 10 hours of polymerization. We believe that the molecular weight of the polymers formed inside the nanopores is limited due to the geometrical constraints. Water uptake of the pore-filled membranes in shown in Figure 6. The sintered polymer-modified membranes did not swell in the course of soaking in water.

Both pore-filled membranes showed similar proton conductivities which steeply increased with relative humidity (Figure7), starting at $0.00001 \mathrm{~S} \mathrm{~cm}^{-1}$ and 0.00002 $\mathrm{S} \mathrm{cm}^{-1}$ for $362 \mathrm{~nm}$ pSMP and $486 \mathrm{~nm}$ pSSA membranes, respectively, at $30{ }^{\circ} \mathrm{C}$ and $30 \%$ R.H., and reaching a maximum value of $0.021 \mathrm{~S} \mathrm{~cm}^{-1}$ at $30{ }^{\circ} \mathrm{C}$ and $\sim 90 \%$ R.H. for both membranes. The proton conductivity for both membranes at $60 \%$ R.H. increased gradually with increasing temperaturefrom $\sim 0.004 \mathrm{~S} \mathrm{~cm}^{-1}$ at $30{ }^{\circ} \mathrm{C}$ to $\sim 0.008 \mathrm{~S} \mathrm{~cm}^{-1} 85^{\circ} \mathrm{C}$, and then decreased to $\sim 0.004 \mathrm{~S} \mathrm{~cm}^{-1}$ at $105{ }^{\circ} \mathrm{C}$. Based on other conductivity measurements at temperatures higher than $85^{\circ} \mathrm{C}$, we conclude that this drop in conductivity resulted from the membrane dehydration and not from the polymer degradation.

We varied the amount of SPM monomer in copolymers filling the colloidal nanopores from $0 \%$ to $100 \%$ by using a second, non-sulfonated monomer (EEMA) and changing the SPM/EEMA monomer ratio. We used NMR and GPC of copolymers prepared in solution in order to confirm the composition and molecular weight of the copolymers. When equal amounts of SPM and EEMA monomers were copolymerized, ${ }^{1} \mathrm{H}$ NMR spectra of the copolymers showed characteristic peaks at $2.9 \mathrm{ppm}\left(-\mathrm{O}-\mathrm{CH}_{2}-\right.$ in SPM fragment) and 3.6-3.7 ppm $\left(-\mathrm{CH}_{2}-\mathrm{O}-\mathrm{CH}_{2}-\right.$ in EEMA fragment). Integration of these peaks gave the molar ratio of SPM/EEMA monomers in copolymer as 1.02, closely matching the initial monomer ratio. According to the GPC analysis, molecular weights of the samples after 1, 2 and 24 hours of copolymerization were 25,41 and $45 \mathrm{kDa}$, respectively. We prepared copolymers using $75 \mathrm{~mol} \%$ and $25 \mathrm{~mol} \% \mathrm{SPM}$, and found the ${ }^{1} \mathrm{H}$ NMR peak integration for to be $3.15: 1$ and 1:3.07, respectively. Based on these results, we concluded that the polymerization rates of SPM and EEMA monomers are similar, which allowed controlling the content of SPM monomers in the copolymers and thus the sulfonic acid group content by varying the ratio of the monomers participating in the polymerization.

We observed a sigmoidal dependence of the proton conductivity on mol\% of SPM monomer in copolymer brushes (Figure 8A). There is a low conductivity region at $20-40 \%$ sulfonic acid group content. Increasing the sulfonic acid group content to $50 \%$ leads to a four-fold increase in proton conductivity. In the range of $50-75 \%$ sulfonic acid group content the proton conductivity grows further. Finally, increasing sulfonic acid group content from 75 to $100 \%$ causes only $\sim 20 \%$ increase in the proton conductivity. We believe that ion-rich clusters in our porefilled membranes become connected to each other when the sulfonated monomer constitutes $50-60 \%$ of the copolymer.

We measured the open circuit voltage (OCV) for the fuel cells made with pore-filed membranes prepared as described above. We varied the sulfonic acid group content andrecordedthe OCV dependence on sulfonic acid group content (Figure 8B). In low and medium range (up to 60\%) OCV dependence on sulfonic group content was similar to that of the proton conductivity. However, in the case of $100 \%$ sulfonated monomer content, the voltage was smaller compared to that for the membranes with $75 \%$ and $60 \%$ SPM content. We hypothesized that this is due to the interplay between the proton conductivity and methanol permeability of the membranes. ${ }^{16}$ We measured the average methanol uptake (directly related to the methanol crossover) and found that for the membranes with less than $60 \%$ SPM monomer content it was $5 \%$, while for the membranes with $>75 \%$ SPM content the methanol uptake was significantly higher, at $13-17 \%$. Since the proton conductivity did not increase significantly above $75 \%$ SPM content, the OCV of the fuel cells using membranes pore- 
filled with $75-100 \%$ pSPM wassmaller due to the increased methanol crossover.

\section{Conclusions}

We demonstrated that membranesprepared by filling the pores in sintered colloidal membranes with sulfonated polymer brushespossess high proton conductivity $(0.021 \mathrm{~S}$ $\mathrm{cm}^{-1}$ at $30{ }^{\circ} \mathrm{C}$ and $\sim 90 \%$ R.H.) and mechanical stability.They contain a continuous network of tunable nanopores, theirwater retention and polyelectrolyte brush structure can be easily controlled.Thus, we believe that these membranes constituteapromising new proton conductive material.

We also prepared proton conductive membranes by selfassembly of silica nanospheres modified with poly(3-sulfopropylmethacrylate) and poly(stryrenesulfonic acid) brushes of different length, and by pore-filling of sintered SNP membranes with the same polymer brushes. The selfassembled membranes showed a higher conductivity (0.052 at $30{ }^{\circ} \mathrm{C}$ and $90 \%$ R.H.) and significantly higher water uptake compared to the pore-filled membranes, which is likely the result of the higher water and sulfonic group content. However, despite the ease of their preparation, self-assembled PBNP membranes are inferior for practical purposes compared to the pore-filled PBNP membranes because of their poor mechanical properties at high water content.

\section{Acknowledgments}

This work was supported the National Science Foundation (CHE-1213628) and by the NSF MRSEC (DMR-1121252) Seed Grant.

\section{References}

[1] Winter, M.; Brodd, R. J. Chem. Rev. 2004, 104, 4245-4269.

[2] Zawodzinski, T. A. Jr.; Springer, T. E.; Uribe, F.; Gottesfeld, S. Solid State Ionics, 1993, 60, 199-211.

[3] Cappadonia, M.; Erning, J. W.; Stimming, U.J. Electroanal. Chem.1994, 376, 189-193.

[4] K. A. Mauritz and R. B. Moore, Chem. Rev., 2004, 104, 4535-4585.

[5] Yeo, R. S.; Cheng, C. H. J. Appl. Polym. Sci.1986, 32, 5733-5741.

[6] Hickner, M. A.; Ghassemi, H.; Kim, Y. S.; Einsla, B. R.; McGrath, J. E. Chem. Rev.2004, 104, 4587-4611.

[7] Li, Q.; He, R.; Jensen, J. O.; Bjerrum, N. J. Chem. Mater.2003, 15, 4896-4915.

[8] Yeo, S. C.; Eisenberg, A. J. Appl. Polym. Sci.1977, 21, 875-898.

[9] Dresselhaus, M.; Crabtree, G.; Buchanan, M. Basic Research Needs for the Hydrogen Economy. A Report on the Basic Energy Sciences Workshop on Hydrogen Production, Storage, and Use. 2004, Argonne National Laboratory, US Department of Energy: Chicago. p. 1-178.

[10] Gary, F. M. Polymer Electrolytes; Royal Society of Chemistry: Cambridge, 1997.

[11] Costamagna, P.; Yang, C.; Bocarsly, A. B.; Srinivasan, S. Electrochim. Acta2002, 47, 1023-1033.

[12] Valle, K.; Belleville, P.; Pereira, F.; Sanchez, C. Nature Mater. 2006, 5, 107-111.

[13] Stöber, W.; Fink, A.; Bohn, E. J. Colloid Interface Sci. 1968, 26, 62 69.

[14] A. K. Bohaty, J. J. Smith and I. Zharov, Langmuir, 2009, 25, 30963101.

[15] A. Khabibullin and I. Zharov, ACS Appl. Mater. Interfaces2014, 6,7712-7718.

[16] X. Li, E. P. L. Roberts and S. M. Holmes, J. Power Sources, 2006, $154,115-123$. 